\title{
A Case Report
}

\section{CONGENITAL SYMPTOMATIC CYTOMEGALOVIRUS INEFCTION}

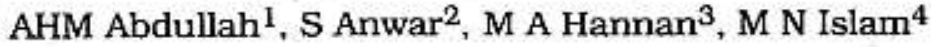

\section{Introduction.}

Cytomegalovirus (CMV) infection is the commonest cause of congenital infection in USA ${ }^{1}$ and Europe ${ }^{2}$. Congenital CMV infection becomes symptomatic only rarely and when it does, it involves multiple organ-systems causing a high rate of morbidity and mortality. There is no specific treatment for this diseae. We report a case of congenital symptomatic cytomegalovirus infection with multi-system involvement in a 50 day old baby.

\section{Case report}

A fifty day old female baby was admitted with complaints of gradual distension of abdomen since birth, convulsion for twenty days and fever for fifteen days. Abdominal distension was not associated with vomiting or constipation. Convulsion was initially generalized but later became focal involving the muscles of the face, eyelids and sometimes of the limbs and used to occur 5-8 times daily. Fever was irregular and occasionally high grade, not associated with chill, rigor or vomiting.

Before admission, she was under the treatment of a Paediatric consultant. Fever subsided without any subsidence of abdominal distension or convulsion, for which the patient was then referred to IPGMR.

On examination, the baby was ill looking, lethargic, moderately pale, afebrile, anicteric having hepatomegaly $(8 \mathrm{~cm}$, firm, smooth, non tender) and splenomegaly (6 cm, firm, smooth) but no lymphadenopathy (FIg-1). There was enophthalmos, microphthalmos and microcornea. Head circumference was normal but both anterior and posterior fontanelles were widely open with sutural diastasis. Convulsion was found to be focal as twitching of musoles. There were no signs of meningeal irritation but muscle tone was increased with extensor plantar response and exaggerated tendon reflexes on both sides. Fundoscopy revealed no abnormality and hearing was normal.

1. Abu Hasan Md. Abdullah MBBS, Asstt. Registrar,

2. Sayeeda Anwar MBBS. Asstt. Registrar,

3. Md. Abdul Hannan MBBS, FCPS, Associate Professor,

4. Md. Nurul Islam MBBS, FCPS, Professor \& Head Department of Child Health, IPGMR. Dhaka. 
Investigations showed anaemia (Hb-50\%), lymphocytic leucocytosis (total WBC count $18000 / \mathrm{mm}^{3}$, differential count $-\mathrm{P}_{20} \mathrm{~L}_{68} \mathrm{M}_{8} \mathrm{E}_{4}$ ), normal platelet count, raised serum GPT (54 U/L) and normal serum bilirubin and alkaline phosphatase. Serum IgG against CMV was positive. CSF analysis showed pleocytosis with high protein and globulin content (cell count $15 / \mathrm{mm}^{3}$, protein $1200 \mathrm{mg} / \mathrm{dl}$ ). Urine culture for CMV could not be done due to lack of facilities. Ultrasonogram of brain revealed hydrocephalus with swollen thalamic and parietal regions. CT scan of brain showed multiple calcified lesions in periventricular regions of both hemispheres, gross dilatation of all ventricles with compressed cerebrum and midline shift to right side (Fig-2).

The baby was diagnosed as a case of congenital cytomegalovirus infection with multi-system involvement and was treated with antibiotics and anticonvulsants. There was no significant improvement except for convulsion after one month of treatment.

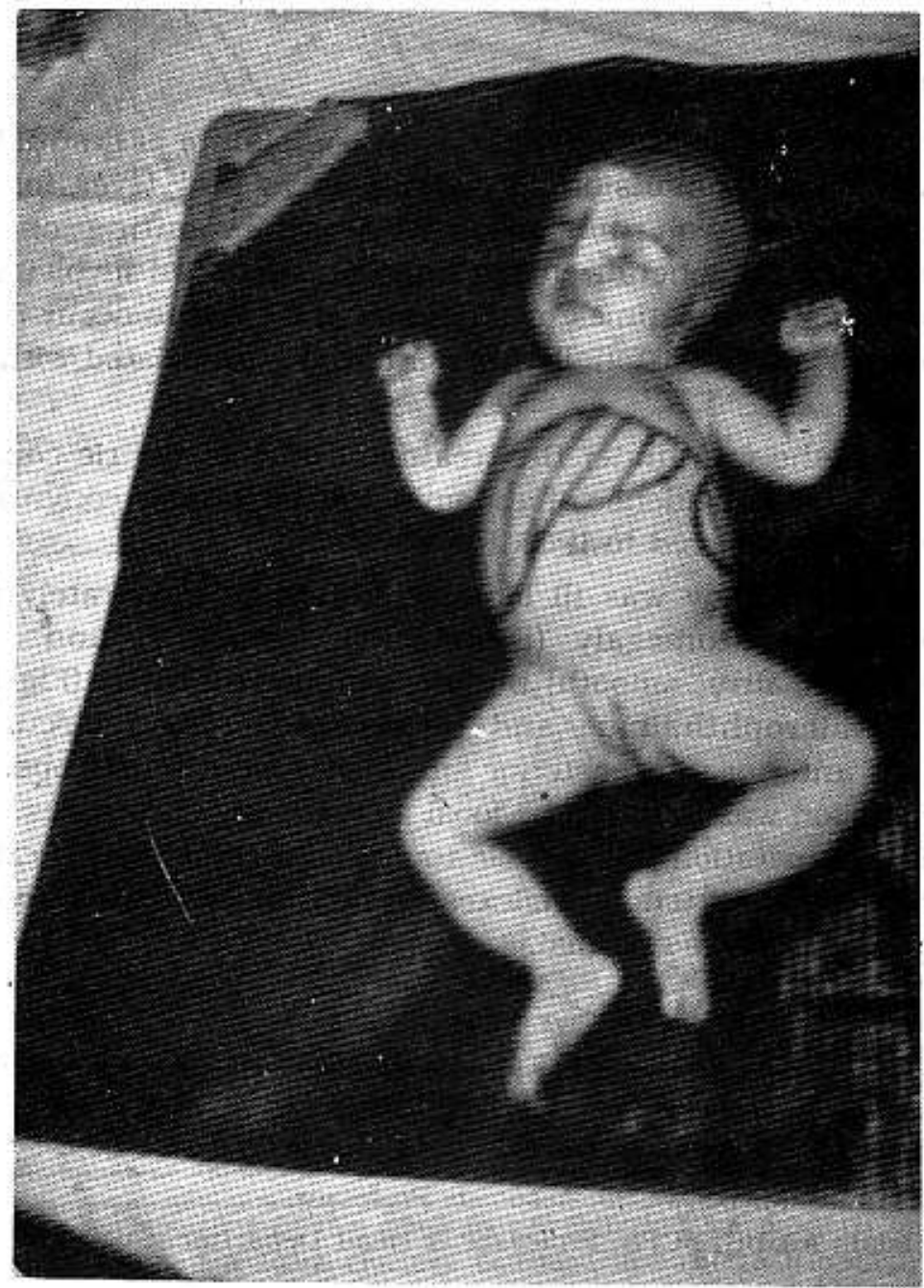




\section{Discussion}

Cytomegalovirus infection is a leading cause of congenital infection. The virus produces characteristic intranuclear and intracytoplasmic inclusion bodies and an increased cell size (cytomegalic). It may be transmitted transplacentally during primary, and less often in recurrent infection of mother. The infection may also be acquired through genital secretion, breast milk or by blood transfusions. Patient may secrete virus in urine and respiratory tract for many months. In about $40 \%$ of primary infection of mother, the foetus will be infected (3-4 per thousand births ${ }^{2}$ ) but most of them will remain asymptomatic. Congenital, symptomatic, multi-organ system cytomegalic inclusion disease is present at birth in less than $10 \%$ and become apparent later in about $25 \%$ of infected infants ${ }^{3}$. When symptomatic, the main clinical features are microcephaly, hydrocephalus, microphthalmia, retinopathy, anaemia, thrombocytopenia, conjugated hyperbilirubinaemia, hepatitis, hepatosplenomegaly, petechial rash, cerebral calcification, deafness, psycomotor retardation and meningoencephalitis. Diabetes insipidus has also been reported ${ }^{4}$. The cerebral calcifications are typically periventricular. Involvement of central nervous system results in most serious sequelae ${ }^{5.6}$. Our patient had cerebral calcification, hydrocephalus and encephalopathy (Seizures, CSF pleocytosis \& elevated protein).

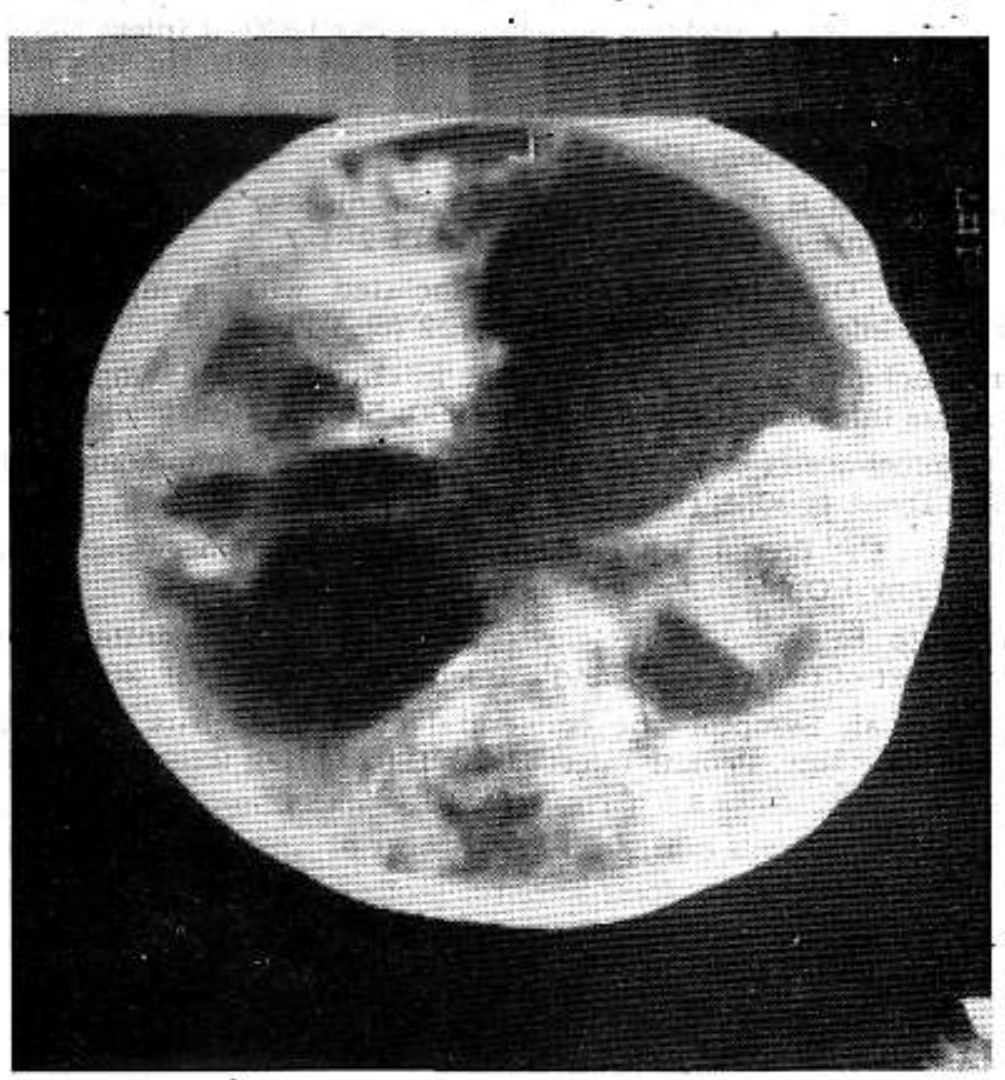


Prior to 1950, cytomegalic inclusion disease was recognized as a fatal disease of infancy in which the diagnosis was invariably made at autopsy ${ }^{2}$. But now-a-days. even prenatal diagnosis can be made by detecting CMV- specific antibody in mother's serum or by virus culture of amniotic fluid ${ }^{7}$. Congenital or neonatal CMV infection can be diagnosed by virus culture of urine, saliva or throat washings, by detection of CMV antigen in urine and saliva of CMV-specific IgM in serum. A stable or rising titre of IgG also supports a diagnosis.

There is no specific therapy for congenital cytomegalovirus infection. The role of Ganciclovir in neonate is unknown, though elimination of virus-shedding in urine without clinical improvement has been reported ${ }^{8}$. Hyperimmune CMV globulin holds prospect especially in immunocompromised hosts. The outcome of symptomatic congenital CMV infection is always poor, with significant mortality and morbidity 2.9 and it is poorest among patients with CNS involvement 5,6 . Psycomotor retardation, hearing loss and learning disabilities frequently occur in this group.

\section{References}

1. Fowler KB, Stagno S, Pass RF, Maternal age and congenital cytomegalovirus infection: screening of two diverse newborn population, 1980-1990. J Infect Dis 1993; I68 (3): 552-6.

2. Compbell AGM, McIntosh N. Forfar and Arneil's Textbook of Paediatrics, 4th edition 1992. Churchill Livingstone, Uk: 314-15.

3. Nardiello S, Galanti B. Congenital cytomegalic disease : current possibilities and prospects of prevention. Clin Ter 1990: 134(5) : 281-7.

4. Mena $W$ et al. Diabetes insipidus associated with symptomatic congenital cytomegalovirus infection. J Pediatr 1993: 122 (6): 911-3.

5. Bale JF Jr, Blackman JA, Sato Y. Outcome in children with symptomatic congenital cytomegalovirus infection. J Child Neurol 1990; 5(2): 131-6.

6. Stagno S. Significance of cytomegalovirus infection in pregnancy and early childhood. Pediatr Infect Dis J 1990; $9(10): 763-4$.

7. Grose $\mathrm{C}$, Weiner $\mathrm{CP}$. Prenatal diagnosis of congenital cytomegalovirus infection : two decades later. Am J Obstet Gynecol 1990: 163(2): 447-50.

8. Attard Montalto SP et al. Ganciclovir treatment of congenital cytomegalovirus Infection: a report of two cases. Scand J Infect Dis 1993:25 (3): 385-8.

9. Boppana SB et al. Symptomatic congenital cytomegalovirus infection : neonatal morbldity and mortality. Pediatr infect Dis J 1992: 11(2): 93-9. 